\title{
45. IRIDIUM AND OTHER ELEMENT DISTRIBUTIONS, MINERALOGY, AND MAGNETOSTRATIGRAPHY NEAR THE CRETACEOUS/TERTIARY BOUNDARY IN HOLE 761C 1
}

\author{
Robert Rocchia, ${ }^{2}$ Daniel Boclet, ${ }^{3}$ Philippe Bonté, ${ }^{2}$ Laurence Froget, ${ }^{2}$ Bruno Galbrun, ${ }^{4}$ Célestine Jéhanno, ${ }^{2}$ \\ and Eric Robin ${ }^{2}$
}

\begin{abstract}
Samples from Ocean Drilling Program Hole 761C, collected on both sides of the Cretaceous/Tertiary boundary have been analyzed for their chemical and mineralogical content. The sediment consists of nannofossil ooze with variable amounts of clay. The boundary is marked by a color change associated with a nearly step-like decrease of the carbonate fraction. Paleomagnetic data and the drop of the carbonate content indicate that a strong reduction of the sedimentation rate occurred at the boundary and persisted for million of years. An iridium anomaly of $80 \mathrm{ng} / \mathrm{cm}^{2}$, together with overabundances of $\mathrm{Cr}$ and $\mathrm{Fe}$, are found in close coincidence with the planktonic crisis. These enrichments can be explained by the infall of $=0.16 \mathrm{~g} / \mathrm{cm}^{2}$ of Cl-like chondritic material. Co and Ni enrichments and a great quantity of Ni-rich magnetites are also observed in the basal Danian. These elements and minerals excepted, the composition of the insoluble fraction appears to be nearly unchanged across the boundary. Chemical and mineralogical observations support a cosmic origin for the Cretaceous/Tertiary event but do not reveal the presence of any significant impact ejecta.
\end{abstract}

\section{INTRODUCTION}

Since the discovery by Alvarez et al. (1980) of an overabundance of iridium commonly attributed to a cosmic event, the number of sections exhibiting the same anomaly has increased to nearly 100 . Although most complete sections systematically show a platinum group element abundance anomaly, they usually differ from one another because of local sedimentation conditions. Many marine Cretaceous/Tertiary $(\mathrm{K} / \mathrm{T})$ sites, especially those located in western Europe, were formed in a hemipelagic or a neritic environment and, therefore, were strongly influenced by the proximity of continents. The study of the boundary sequence at sites that formed under different depositional conditions is a good way to better understand the influence of the various sedimentary sources. Leg 122 offers the opportunity to explore an area where few $\mathrm{K} / \mathrm{T}$ boundaries have been found so far. Hole $761 \mathrm{C}$ was cored on the Wombat Plateau, the most northern extension of the Exmouth Plateau, northeast of Australia. A complete core across the $\mathrm{K} / \mathrm{T}$ boundary was recovered from a sedimentary sequence containing little terrigenous material that was formed in a pelagic environment (Haq, von Rad, O'Connell, et al., 1990). The Maestrichtian part of the core consists of white ooze of nannofossils; the Danian portion is richer in clay. There is no distinct clay layer at the boundary (see core photograph in Haq, von Rad, O'Connell, et al., 1990), which is marked by only a slight color change from white to light brown and green. This paper reports on the Ir enrichment at the $\mathrm{K} / \mathrm{T}$ boundary in Hole $761 \mathrm{C}$. It also includes discussions of paleomagnetic measurements across the boundary and preliminary results of our search for debris and other traces of cosmic origin.

\footnotetext{
1 von Rad, U., Haq, B. U., et al., 1992. Proc. ODP, Sci. Results, 122: College Station, TX (Ocean Drilling Program).

2 Centre des Faibles Radioactiviés, Laboratoire Mixte CEA-CNRS, Avenue de la Terrasse, 91198 Gif-sur-Yvette Cedex, France.

${ }^{3}$ Service d'Astrophysique, CEN Saclay, 91191 Gif-sur-Yvette Cedex, France.

${ }^{4}$ Université de Paris VI, Département de Géologie Sédimentaire, URA CNRS 1314, 4 place Jussieu, 75252 Paris Cedex, France.
}

\section{ANALYTICAL METHODS}

\section{Sampling}

Samples were provided in two sets. The first one continuously covers the interval between Sample 122-761C-3R-3, $52-53 \mathrm{~cm}$, and Sample 122-761C-3R-3, 71-72 cm, with a 1-cm spacing. It was supposed to include the $\mathrm{K} / \mathrm{T}$ boundary which, according to the study of calcareous nannofossils populations, was placed around interval $122-761 \mathrm{C}-3 \mathrm{R}-3,67-68 \mathrm{~cm}$. The rest of the section was more loosely sampled with a $5-\mathrm{cm}$ step. Analyses of this first set of samples shows that the Ir maximum concentration is actually located between Sample 122761C-3R-3, 71-72 cm, and Sample 122-761C-3R-3, 80-81 cm, well below the expected position. Then, additional samples taken in a $10-\mathrm{cm}$-long subcore covering the interval $122-761 \mathrm{C}$ $3 R-3,70-80 \mathrm{~cm}$, were analyzed. The relative position of the two sets of samples was controlled thanks to the sharp color change observable in this subcore and in Sample 122-761C$3 \mathrm{R}-3,75-76 \mathrm{~cm}$. In view of the Ir distribution, which extends beyond the limits of the sampled interval, additional distant samples, some of them first used for paleomagnetic studies, were included in our routine for background determination.

\section{Iridium and Other Elements}

Elemental analyses were carried out by instrumental neutron activation analysis (INAA) of whole-rock samples. About $100 \mathrm{mg}$ of ground and well-homogenized sample were dried, then sealed in a pure quartz vial. They were irradiated for a few hours in the $2 \times 10^{14} \mathrm{n} / \mathrm{cm}^{2} / \mathrm{s}$ neutron beam of the Osiris reactor at the Pierre Süe Laboratory, Saclay. Calibration standards are a piece of aluminium loaded with $5 \mathrm{ppm}$ of Ir and USGS MAG1 for other elements. Iridium was counted with a $\gamma-\gamma$ spectrometer detecting, without Compton rejection device, the 316-468 $\mathrm{keV} \gamma$-ray coincidence resulting from the decay of ${ }^{192} \mathrm{Ir}$. The sensitivity is such that concentrations as low as 50 picograms per gram $(\mathrm{pg} / \mathrm{g})$ can be significantly measured ( 3 standard deviation confidence level) after a 1-day accumulation time. Such good accuracy partly results from the improved performance of the $\gamma-\gamma$ spectrometer over usual devices and partly from the low clay content of most samples. 
In order to determine Ir backgrounds as precisely as possible, several distant samples (more than $1 \mathrm{~m}$ away from the boundary) were counted together over a weekend. This permitted us to lower the sensitivity by a factor of 3 to 5 . Concentrations of other elements were measured with a single high-purity $\mathrm{Ge}$ detector.

\section{Paleomagnetism}

Minicores oriented with respect to the upcore direction were drilled perpendicular to the axis of the split core at various depth intervals. Nine samples were taken in Core $122-761 \mathrm{C}-2 \mathrm{R}$ and 16 in Core $122-761 \mathrm{C}-3 \mathrm{R}$. All the measurements were made with the 3 -axis RS-01 (LETI/CEA) cryogenic magnetometer of the Département de Géologie Sédimentaire, Université Paris VI. The sensitivity of the instrument is such that it is possible to determine magnetization directions for samples with natural remanent magnetization (NRM) intensity as weak as $2 \times 10^{-5} \mathrm{~A} / \mathrm{m}$ with a good precision. Usual alternating field (AF) and thermal demagnetization techniques were used to isolate primary remanence components. The characteristic remanent magnetization (ChRM) directions were computed by applying the threedimensional least-square line fit method of Kirschvink (1980).

\section{Mineralogy}

The determination of the mineral components was made by means of X-ray powder diffraction technique using a Philips goniometer equipped with a copper anode tube. Analyses were carried out on whole-rock powdered samples and some on the insoluble residues after dissolution of the carbonate fraction with $0.1-\mathrm{N}$ acetic acid. We also measured major and minor element concentrations on compacted pellets with a LINK X-ray energy dispersive spectrometer (EDS) attached to a JEOL scanning electron microscope (SEM). Magnetically separated particles were analyzed with the SEM-EDS device.

The carbonate fraction was estimated from the $\mathrm{Ca}$ content measured both by INAA and EDS analysis. On a few samples, the process was checked by weighing the residue after carbonate dissolution.

\section{RESULTS}

\section{Iridium and Other Siderophile Elements}

The Ir abundance distribution is plotted in Figure 1 as a function of sample depth measured in meters below seafloor (mbsf). It is characterized by a sharp increase of the concentration in Sample 122-761C-3R-3, 75-76 cm (172.46 mbsf), in close coincidence with the color change observed at this level. The concentration remains nearly constant over a plateau of a few centimeters, followed by a tail extending over several tens of centimeters. Besides this major peak and two small bumps in the upward-extending tail, there is no outstanding sharp feature in the distribution. The main Ir anomaly is superimposed on a sort of shoulder that extends symmetrically on both sides of the boundary over a total thickness of $2 \mathrm{~m}$ of core. Estimates of the background concentrations, derived from the counting of most distant samples, have only a marginal statistical significance: $5 \pm 2(1-\sigma) \mathrm{pg} / \mathrm{g}$ for the Maestrichtian and $22 \pm 8(1-\sigma) \mathrm{pg} / \mathrm{g}$ during early Danian. To estimate the total mass of anomalous $\mathrm{Ir}$ at the $\mathrm{K} / \mathrm{T}$ boundary we have integrated the Ir distribution multiplied by the average density $(d=1.35)$ over the interval of $2 \mathrm{~m}$ where the Ir concentration is significantly higher than the background. The total Ir flux is about 80 nanograms per square centimeter $\left(\mathrm{ng} / \mathrm{cm}^{2}\right)$, representing a mass of $0.16 \mathrm{~g} / \mathrm{cm}^{2}$ of $\mathrm{Cl}$-like chondritic material, if a meteoritic source is supposed. The shoulder represents about $40 \%$ of the total Ir infall.

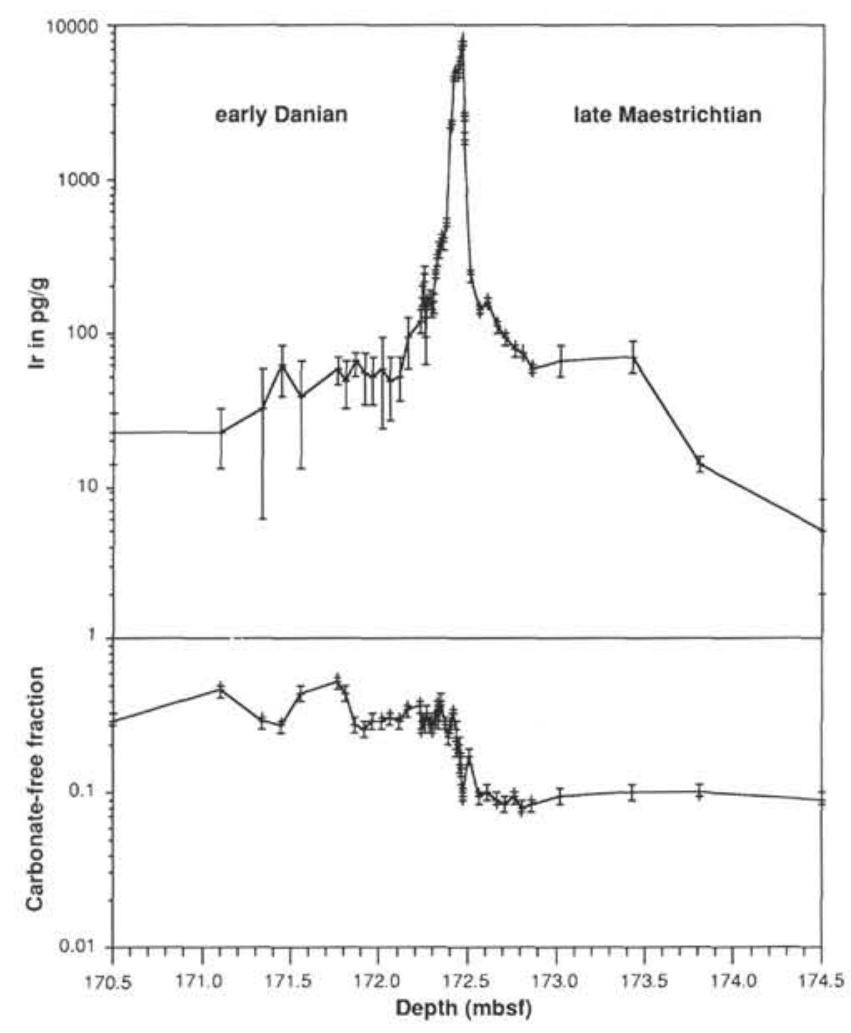

Figure 1. Distribution of $\mathrm{Ir}$ and carbonate-free fraction across the $\mathrm{K} / \mathrm{T}$ boundary in Hole 761C. The Ir backgrounds in the Maestrichtian (175 to $177 \mathrm{mbsf}$ ) and in the Danian (170 to $171 \mathrm{mbsf}$ ) are $5 \pm 2 \mathrm{pg} / \mathrm{g}$ and 22 $\pm 8 \mathrm{pg} / \mathrm{g}$, respectively. Error bars are \pm 1 standard deviation.

As far as other siderophile elements are concerned, $\mathrm{Cr}, \mathrm{Fe}$, $\mathrm{Co}$, and $\mathrm{Ni}$ show significant excesses over their concentration in the terrigenous clay fraction. This is illustrated by the graphs in Figure 2 that display the abundances of these elements normalized to Sc. This element, as well as Th, is an excellent trace element indicator for the terrigenous clay component. The development of all these anomalies are rapid and somewhat shorter than the evolution of the Ir distribution. The excess of Sc-normalized abundances of $\mathrm{Cr}, \mathrm{Fe}, \mathrm{Co}$, and $\mathrm{Ni}$ is determined in each sample by subtracting the terrigenous background level shown as dotted lines on Figure 2. The total elemental excess is then obtained by integrating the Scnormalized excess values after multiplication by the Sc concentration, which varies from sample to sample. Doing that, we obtain an integrated $\mathrm{Cr}$ excess of $400 \mu \mathrm{g} / \mathrm{cm}^{2}$ that can be explained by the fall of $0.15 \mathrm{~g} / \mathrm{cm}^{2}$ of $\mathrm{Cl}$-like chondritic matter. A similar value of $0.14 \mathrm{~g} / \mathrm{cm}^{2}$ is derived from the integrated excess of $\mathrm{Fe}\left(26 \mathrm{mg} / \mathrm{cm}^{2}\right)$. Co and $\mathrm{Ni}$, elements that are always associated with Ir in extraterrestrial matter, are not characterized by strong anomalies. The integrated excesses of $\mathrm{Co}$ $\left(5.4 \mu \mathrm{g} / \mathrm{cm}^{2}\right)$ and $\mathrm{Ni}\left(65 \mu \mathrm{g} / \mathrm{cm}^{2}\right)$ are respectively consistent, with only $0.01 \mathrm{~g} / \mathrm{cm}^{2}$ and $0.006 \mathrm{~g} / \mathrm{cm}^{2}$ of $\mathrm{Cl}$-like chondritic matter.

\section{Rare-Earth Patterns and Other Elements}

Whole-rock rare-earth element patterns (Fig. 3) normalized to chondrites are similar from the Maestrichtian (Fig. 3C) to the Danian (Fig. 3A) with no marked difference at the $\mathrm{K} / \mathrm{T}$ boundary (Fig. 3B) defined as coinciding with the maximum Ir enrichment. The slight $\mathrm{Ce}$ depletion indicates that a small amount of rare-earth elements was derived from seawater. This anomaly is present all along the core with no detectable 


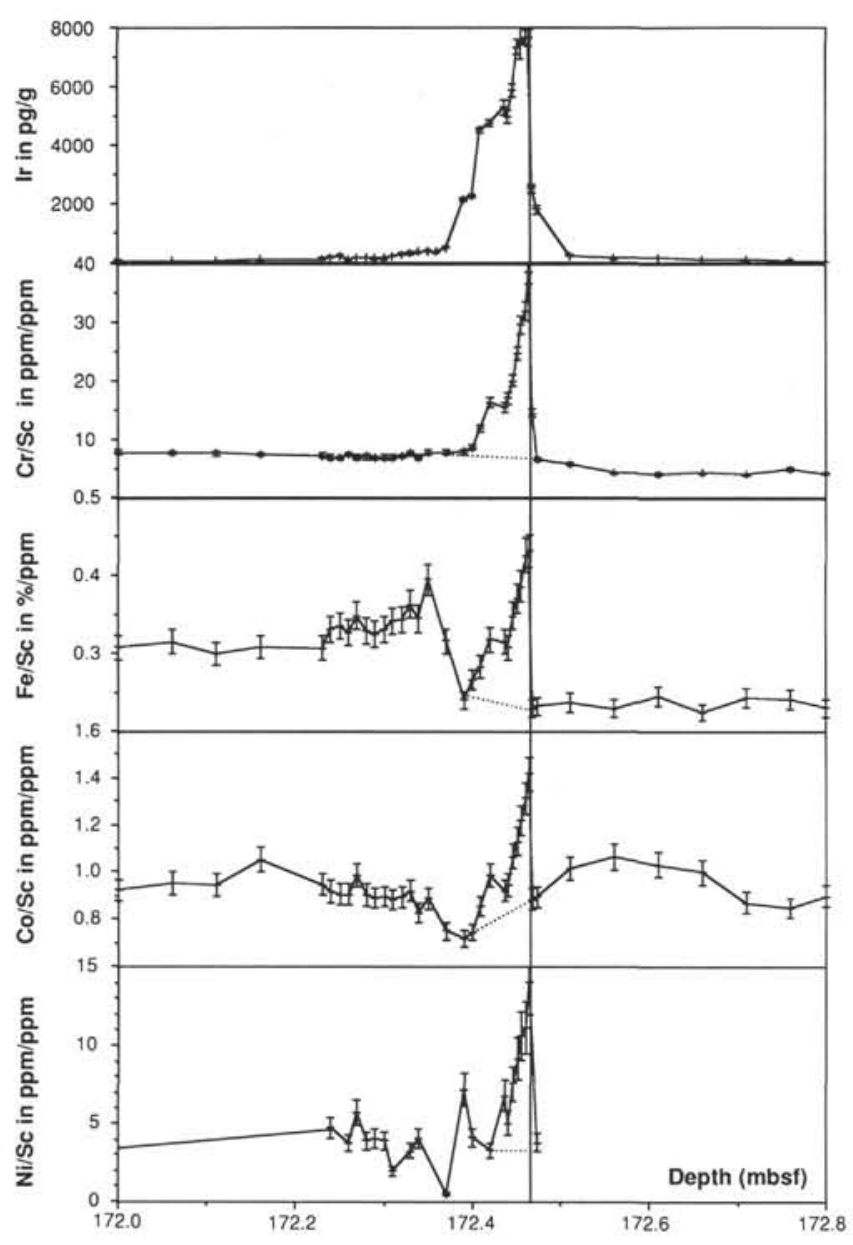

Figure 2. Distribution of $\mathrm{Ir}$ and Sc-normalized abundances of $\mathrm{Cr}, \mathrm{Fe}$, $\mathrm{Co}$, and $\mathrm{Ni}$ vs. stratigraphic depth (mbsf). The distributions of $\mathrm{Cr}, \mathrm{Fe}$, $\mathrm{Co}$, and $\mathrm{Ni}$ are well correlated and more narrow than the $\mathrm{Ir}$ anomaly.

change at the $\mathrm{K} / \mathrm{T}$ boundary (Fig. 3D). However, the $\mathrm{La} / \mathrm{Yb}$ ratio which is about $10: 12$ in the Maestrichtian, regularly increases from $\mathrm{K} / \mathrm{T}$ boundary up to a maximum of 15 occurring $7-8 \mathrm{~cm}$ above it, and then returns to the initial value of 10:12 in the rest of the Danian.

$\mathrm{Th}, \mathrm{Cs}, \mathrm{Hf}, \mathrm{Ta}, \mathrm{Sc}$, and $\mathrm{Al}$ (Table 1) all follow the evolution of the clay fraction.

\section{Mineralogy}

All whole-rock X-ray-diffraction spectra are dominated by calcite (Figs. 4A, 4B, 4C, and 5). Three other phases appear which are smectite and clinoptilolite with little quartz. In addition to these phases, some samples located near siliceous concretions contain abundant opal-CT. We note that the same mineralogic phases are continuously observed from the Cretaceous to the Paleocene with no change at the $\mathrm{K} / \mathrm{T}$ boundary (compare spectrum in Fig. 4B with 4A and 4C). Clinoptilolite (PI. $1 \mathrm{~A}$ and $1 \mathrm{~B})$ and smectite are authigenic phases that likely arise from the alteration of volcanic glasses. We have no reason to suspect that, at the very $\mathrm{K} / \mathrm{T}$ boundary, these minerals would have a different origin. Quartz is detrital whereas opal-CT has a biogenic origin. Spectra of X-ray diffraction carried out on the insoluble fraction only (Fig. 5) do not reveal other phases than those previously observed in whole-rock samples and show that smectite and clinoptilolite are fairly well crystallized.

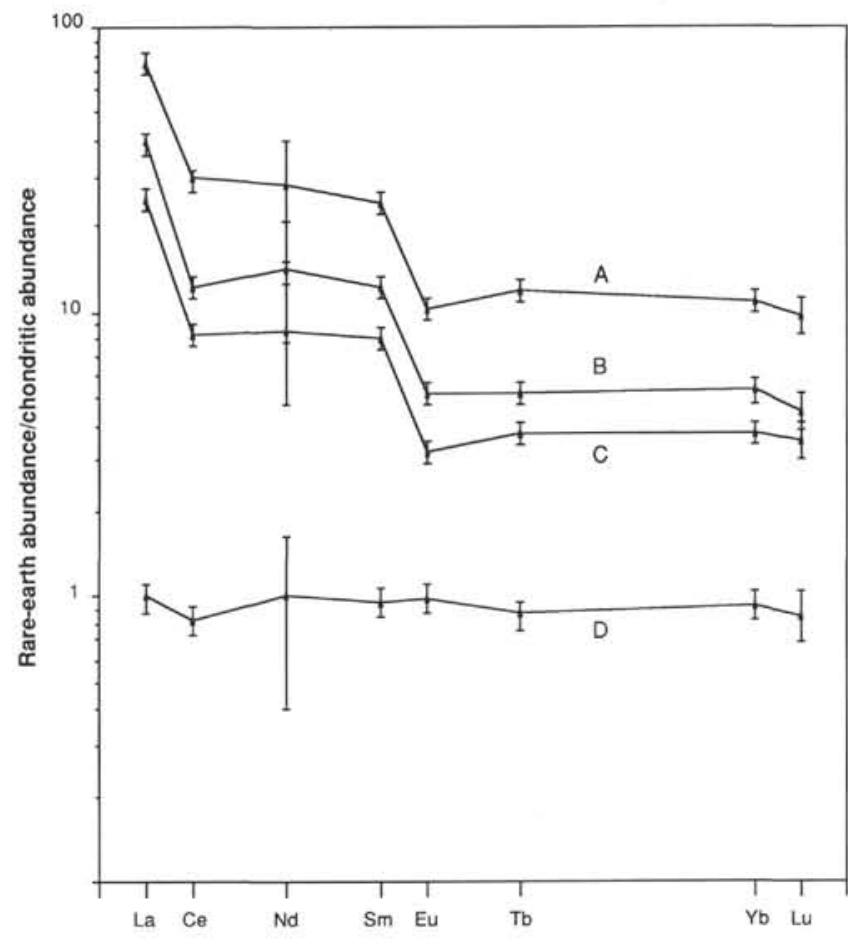

Figure 3. Rare-earth elements abundances normalized to chondrites as a function of atomic number. A. Danian, $20 \mathrm{~cm}$ above the boundary; Sample 122-761C-3R-3, 55-56 cm (172.26 mbsf). B. K/T boundary defined as the maximum of Ir enrichment; Sample 122761C-3R-3, 75-76 cm (172.464 mbsf). C. Maestrichtian, $20 \mathrm{~cm}$ below the boundary; Sample 122-761C-3R-3, 95-96 cm (172.66 mbsf). D. $(0.5 \times$ boundary $) /($ Maestrichtian + Danian $)$, normalized to lanthanum. Error bars are \pm 3 standard deviations.

Optical observations and magnetic extraction reveal the existence in the first few centimeters of the Danian of a great quantity of black Ni-rich magnetite crystals. These crystals are often clustered in flat pancakes or lenses (PI. 1C), sometimes exceeding several hundred microns in diameter. These clusters are aligned parallel to the deposition plane and are analogous to flattened spheroids found in some other $\mathrm{K} / \mathrm{T}$ sections and described by Montanari et al. (1983), Smit and Kyte (1984), and Kyte and Smit (1986). The typical composition of magnetites is $5 \%-10 \% \mathrm{Mg}, 2 \%-4 \% \mathrm{Al}, 0.5 \%-2 \% \mathrm{Cr}, 50 \%-55 \% \mathrm{Fe}$, and $2 \%-6 \% \mathrm{Ni}$. Individual crystals are rather large: magnetites exceeding 10-15 $\mu \mathrm{m}$ are common (Pl. 1D). They are embedded in a matrix of clay which is possibly the result of alteration of some original material, probably spherical in shape, that was subsequently flattened during compaction.

A brief microscopic examination of Sample 122-761C-3R-3, $75-76 \mathrm{~cm}$, did not reveal the presence of shocked quartz; however, its absence in this small sample $(<1 \mathrm{~g})$ does not preclude its possible discovery in later investigations of samples from Hole 761C.

\section{Magnetostratigraphy}

Alternating field (AF) and thermal demagnetization both produced good results. An unstable component was removed by heating at $100^{\circ} \mathrm{C}$ or by $5-10 \mathrm{mT}$ AF demagnetization, and a stable component is observed within the $150^{\circ}-400^{\circ} \mathrm{C}$ or $10-30 \mathrm{mT}$ range of demagnetization steps (Fig. 6).

Since borehole cores are not azimuthally oriented, only inclination values are used for interpretation (Table 2). With the exception of two samples of normal polarity (122-761C- 
Table 1. Elemental abundances across the $\mathrm{K} / \mathrm{T}$ boundary in Hole $761 \mathrm{C}$.

\begin{tabular}{|c|c|c|c|c|c|c|c|c|c|c|c|c|c|c|}
\hline $\begin{array}{l}\text { Core, section, } \\
\text { interval }(\mathrm{cm})\end{array}$ & $\begin{array}{l}\text { Depth } \\
\text { (mbsi) }\end{array}$ & $\mathrm{CaCO}$ & $\begin{array}{c}\mathrm{Sr} \\
\mathrm{ppm}\end{array}$ & $\mathrm{Mg}_{\%}$ & ${ }^{A l}$ & $\begin{array}{l}\mathrm{Si} \\
\% \\
\end{array}$ & $\begin{array}{r}\mathrm{Fe}_{\mathrm{O}} \\
\%\end{array}$ & $\begin{array}{l}\mathrm{Sc} \\
\mathrm{ppm}\end{array}$ & $\begin{array}{c}\text { Th } \\
\mathrm{ppm}\end{array}$ & $\begin{array}{c}\mathrm{HI} \\
\mathrm{ppm}\end{array}$ & $\begin{array}{r}\mathrm{Ta} \\
\mathrm{ppm}\end{array}$ & $\begin{array}{r}\text { Cs } \\
\text { ppm }\end{array}$ & $\begin{array}{r}\text { Ro } \\
\mathrm{ppm}\end{array}$ & $\begin{array}{r}\mathrm{Ba} \\
\mathrm{ppm}\end{array}$ \\
\hline \multicolumn{15}{|l|}{$122.761 \mathrm{C}}$. \\
\hline $2 \mathrm{R}-2, \quad 124-125$ & 162.940 & 0.615 & 610 & 0.4 & 0.9 & 15.2 & 0.47 & 1.89 & 1.90 & 0.30 & 0.090 & 0.58 & 12 & 450 \\
\hline 2R-3, $118-119$ & 164.380 & 0.776 & 689 & 0.5 & 1.8 & 6.3 & 1.00 & 3.43 & 3.70 & 0.61 & 0.180 & 1.30 & 21 & 430 \\
\hline $3 R-1,48-49$ & 170.180 & 0.689 & & 1.0 & 2.6 & 9.0 & & & & & & & & \\
\hline $3 R-1,63-64$ & 170.330 & 0.697 & & 0.7 & 2.3 & 9.4 & & & & & & & & \\
\hline $3 R-1, \quad 102-103$ & 170.720 & 0.702 & & 0.9 & 2.2 & 8.3 & & & & & & & & \\
\hline $3 \mathrm{R}-1,140-141$ & 171.100 & 0.532 & & 0.5 & 2.1 & 17.3 & & & & & & & & \\
\hline $3 \mathrm{~A} \cdot 2, \quad 14-15$ & 171.340 & 0.712 & & 0.7 & 2.2 & 8.4 & & & & & & & & \\
\hline $3 \mathrm{~A}-2,25-26$ & 171.450 & 0.727 & & 0.7 & 2.7 & 7.4 & & & & & & & & \\
\hline $3 R \cdot 2, \quad 35 \cdot 36$ & 171.550 & 0.550 & & 1.1 & 4.8 & 11.9 & & & & & & & & \\
\hline $3 R-2, \quad 48-49$ & 171.680 & 0.561 & 604 & 1.0 & 3.4 & 12.8 & 1.90 & 7.13 & 6.80 & 1.30 & 0.360 & 3.10 & 45 & 1730 \\
\hline $3 R-3,5-6$ & 171.760 & 0.473 & 550 & 1.1 & 4.0 & 16.1 & 2.13 & 6.95 & 7.00 & 1.30 & 0.350 & 3.00 & 42 & 1340 \\
\hline $3 \mathrm{~A}-3, \quad 10-11$ & 171.810 & 0.556 & 526 & 0.9 & 3.4 & 13.5 & 1.93 & 6.88 & 7.00 & 1.30 & 0.400 & 3.00 & 45 & 1000 \\
\hline $3 R-3, \quad 15 \cdot 16$ & 171.860 & 0.723 & 665 & 0.6 & 1.9 & 8.2 & 1.59 & 4.93 & 5.00 & 1.00 & 0.270 & 2.00 & 34 & 1080 \\
\hline $3 \mathrm{~A}-3,20-21$ & 171.910 & 0.739 & 672 & 0.6 & 1.9 & 7.5 & 1.22 & 3.82 & 3.60 & 0.76 & 0.220 & 1.50 & 23 & 1100 \\
\hline $3 \mathrm{R}-3,25-26$ & 171.960 & 0.706 & 568 & 0.7 & 2.2 & 8.7 & 1.56 & 5.10 & 5.00 & 1.10 & 0.300 & 2.20 & 33 & 1510 \\
\hline $3 \mathrm{~A} \cdot 3,30-31$ & 172.010 & 0.710 & 632 & 0.6 & 2.1 & 8.5 & 1.40 & 4.55 & 4.40 & 0.90 & 0.250 & 1.90 & 28 & 1120 \\
\hline $3 \mathrm{~A}-3, \quad 35-36$ & 172.060 & 0.694 & 623 & 0.7 & 2.2 & 9.0 & 1.42 & 4.51 & 4.40 & 0.90 & 0.250 & 1.90 & 35 & 1080 \\
\hline $3 \mathrm{~F}-3,40-41$ & 172.110 & 0.711 & 732 & 0.6 & 2.2 & 8.7 & 1.17 & 3.90 & 3.70 & 0.70 & 0.220 & 1.50 & 27 & 1160 \\
\hline $3 \mathrm{R}-3,45 \cdot 46$ & 172.160 & 0.658 & 714 & 0.8 & 2.5 & 9.7 & 1.52 & 4.93 & 4.80 & 1.00 & 0.310 & 1.90 & 39 & 1130 \\
\hline $3 \mathrm{~A}-3, \quad 52-53$ & 172.230 & 0.634 & 750 & 0.8 & 2.8 & 11.0 & 1.77 & 5.76 & 5.40 & 1.20 & 0.290 & 2.20 & 37 & 1450 \\
\hline $3 \mathrm{~A}-3,53-54$ & 172.240 & 0.732 & 616 & 0.7 & 2.0 & 7.6 & 1.75 & 5.27 & 4.70 & 0.80 & 0.200 & 1.60 & 27 & 950 \\
\hline $3 \mathrm{~A}-3,54-55$ & 172.250 & 0.711 & 574 & 0.8 & 2.2 & 8.0 & 1.85 & 5.52 & 4.70 & 0.85 & 0.180 & 1.70 & 26 & 1070 \\
\hline $3 \mathrm{R}-3,55-56$ & 172.260 & 0.727 & 634 & 0.8 & 1.9 & 7.6 & 1.75 & 5.35 & 4.70 & 0.85 & 0.180 & 1.60 & 25 & 1070 \\
\hline $3 \mathrm{~A}-3,56-57$ & 172.270 & 0.668 & 572 & 0.7 & 2.1 & 8.0 & 1.83 & 5.25 & 5.10 & 0.90 & 0.230 & 1.70 & 30 & 1100 \\
\hline $3 \mathrm{R}-3,57-58$ & 172.280 & 0.726 & 600 & 0.7 & 2.1 & 8.0 & 1.67 & 5.08 & 4.40 & 0.80 & 0.200 & 1.50 & 26 & 1110 \\
\hline $3 \mathrm{~A}-3,58-59$ & 172.290 & 0.700 & 593 & 0.8 & 2.3 & 8.5 & 1.75 & 5.38 & 4.70 & 0.80 & 0.190 & 1.70 & 27 & 1020 \\
\hline $3 \mathrm{~A}-3,59 \cdot 60$ & 172.300 & 0.732 & 566 & 0.7 & 2.0 & 7.7 & 1.70 & 5.12 & 4.50 & 0.80 & 0.180 & 1.60 & 28 & 1020 \\
\hline $3 \mathrm{~A}-3,60-61$ & 172.310 & 0.691 & 523 & 0.9 & 2.4 & 8.6 & 1.70 & 4.97 & 4.60 & 0.70 & 0.200 & 1.60 & 27 & 960 \\
\hline $3 \mathrm{~A}-3,61.62$ & 172.320 & 0.703 & 587 & 0.8 & 2.4 & 8.5 & 1.82 & 5.30 & 4.70 & 0.70 & 0.180 & 1.70 & 28 & 940 \\
\hline $3 R-3,62-63$ & 172.330 & 0.633 & 527 & 0.9 & 2.6 & 10.3 & 2.33 & 6.41 & 6.10 & 1.00 & 0.260 & 2.20 & 34 & 1150 \\
\hline $3 R-3,63-64$ & 172.340 & 0.707 & 536 & 0.8 & 2.0 & 8.2 & 1.95 & 5.65 & 4.80 & 0.90 & 0.200 & 1.80 & 28 & 1240 \\
\hline $3 \mathrm{~A}-3, \quad 64-65$ & 172.350 & 0.596 & 495 & 1.0 & 3.0 & 11.6 & 2.60 & 6.58 & 6.40 & 1.10 & 0.250 & 2.30 & 35 & 1520 \\
\hline $3 \mathrm{~A}-3, \quad 66-67$ & 172.370 & 0.716 & 520 & 0.8 & 2.0 & 7.9 & 1.77 & 5.60 & 5.20 & 1.00 & 0.190 & 1.90 & 32 & 1490 \\
\hline $3 \mathrm{~A}-3,68-69$ & 172.390 & 0.768 & 675 & 0.5 & 1.7 & 7.0 & 0.94 & 3.90 & 3.58 & 0.72 & 0.190 & 1.60 & 26 & 280 \\
\hline $3 R-3,69-70$ & 172.400 & 0.726 & 795 & 0.6 & 2.1 & 8.2 & 1.36 & 5.10 & 5.10 & 1.00 & 0.260 & 2.00 & 37 & 370 \\
\hline $3 A-3,70-71$ & 172.410 & 0.699 & 718 & & & & 1.06 & 3.74 & 3.60 & 0.68 & 0.180 & 1.50 & & 340 \\
\hline $3 R-3,71-72$ & 172.420 & 0.679 & 844 & 1.0 & 2.2 & 9.2 & 1.16 & 3.65 & 3.40 & 0.65 & 0.180 & 1.30 & 21 & 265 \\
\hline 3R-3, $72.5-72.8$ & 172.431 & 0.736 & 749 & 0.6 & 2.0 & 7.6 & 1.05 & 3.3 & 2.98 & 0.60 & 0.166 & 1.0 & 20 & 277 \\
\hline $3 \mathrm{~A}-3,73.0-73.3$ & 172.436 & $0.780^{\circ}$ & 694 & 0.5 & 1.6 & 5.8 & 0.91 & 2.97 & 2.38 & 0.51 & 0.135 & 0.8 & 18 & 223 \\
\hline $3 \mathrm{~A}-3,73.5-73.8$ & 172.441 & 0.791 & 783 & 0.6 & 1.5 & 5.9 & 1.07 & 3.07 & 2.61 & 0.53 & 0.142 & 0.9 & 18 & 284 \\
\hline $3 A-3,74.0-74.3$ & 172.446 & 0.826 & 851 & 0.5 & 1.2 & 4.8 & 1.02 & 2.75 & 2.19 & 0.46 & 0.116 & 0.76 & 14 & 293 \\
\hline $3 \mathrm{R}-3,74.5-74.8$ & 172.451 & 0.846 & 880 & 0.5 & 1.1 & 4.2 & 0.94 & 2.43 & 1.91 & 0.39 & 0.096 & 0.63 & 12 & 352 \\
\hline $3 \mathrm{R}-3,75.0 \cdot 75.3$ & 172.456 & & 803 & & & & 0.96 & 2.31 & 1.81 & 0.41 & 0.101 & 0.6 & 10 & 301 \\
\hline $3 \mathrm{R}-3,75.3-75.5$ & 172.459 & 0.870 & 847 & 0.4 & 0.9 & 3.6 & 0.93 & 2.16 & 1.72 & 0.35 & 0.098 & 0.57 & 11 & 334 \\
\hline $3 \mathrm{~A}-3,75.5-75.8$ & 172.461 & 0.850 & 906 & 0.4 & 1.0 & 3.9 & 0.88 & 2.05 & 1.73 & 0.33 & 0.091 & 0.54 & 11 & 351 \\
\hline $3 R-3,75.8-76.0$ & 172.464 & 0.902 & 926 & & & & 0.48 & 1.57 & 1.02 & 0.21 & 0.056 & 0.34 & 6 & 482 \\
\hline $3 \mathrm{~A}-3,76-76.3$ & 172.466 & 0.900 & 997 & & & & 0.36 & 1.49 & 0.96 & 0.19 & 0.049 & 0.31 & 7 & 471 \\
\hline $3 \mathrm{~A}-3,76.3-76.5$ & 172.469 & & 963 & & & & 0.3 & 1.2. & 1.0 & 0.19 & 0.048 & 0.3 & 6 & 446 \\
\hline $3 \mathrm{~A}-3,80-81$ & 172.510 & 0.828 & 995 & 0.2 & 0.5 & 6.5 & 0.2 & 1.18 & 1.10 & 0.20 & 0.050 & 0.3 & 7 & 410 \\
\hline $3 \mathrm{~A}-3,85 \cdot 86$ & 172.560 & 0.906 & 1170 & 0.2 & 0.4 & 2.8 & 0.28 & 1.22 & 1.10 & 0.20 & 0.060 & 0.4 & 5 & 495 \\
\hline $3 \mathrm{R}-3,90-91$ & 172.610 & 0.898 & 1193 & 0.3 & 0.5 & 3.1 & 0.31 & 1.26 & 1.15 & 0.23 & 0.050 & 0.40 & 5 & 480 \\
\hline $3 R-3,95-96$ & 172.660 & 0.908 & $114 \mathrm{C}$ & 0.3 & 0.5 & 2.4 & 0.2 & 1.2 & 11 & 0.20 & 0.050 & 0.4 & 5 & 485 \\
\hline $3 \mathrm{~A}-3, \quad 100-1$ & 172 & 0.9 & 10 & 0. & 0.5 & 2.3 & & 1.2 & $i 1$ & 0.23 & 0.052 & 0.3 & 7 & 48 \\
\hline $3 \mathrm{R}-3, \quad 105-1$ & 172 & & 113 & 0.2 & 0.6 & 2.5 & 0. & & 1. & & & & 8 & 45 \\
\hline $3 R-3,110-111$ & 172.810 & 0.918 & 874 & 0.3 & 0.5 & 2.0 & 0.23 & 1.0 & 0.9 & 0.16 & 0.040 & 0.3 & & 380 \\
\hline $3 R-3,115-116$ & 172.860 & 0.917 & 1012 & 0.3 & 0.5 & 2.0 & 0.28 & 1.19 & 1.10 & 0.18 & 0.050 & 0.38 & 6 & 420 \\
\hline $3 \mathrm{R}-3,130-131$ & 173.020 & 0.905 & & 0.2 & 0.6 & 2.2 & & & & & & & & \\
\hline $3 \mathrm{~A}-4,21-22$ & 173.430 & 0.898 & & 0.2 & 0.6 & 2.6 & & & & & & & & \\
\hline $3 R-4,59-60$ & 173.810 & 0.897 & 1146 & 0.4 & 0.6 & 2.6 & 0.31 & 1.27 & 1.10 & 0.23 & 0.050 & 0.38 & 7 & 460 \\
\hline $3 \mathrm{R}-4,80-81$ & 174.020 & 0.911 & 1140 & 0.3 & 0.5 & 2.2 & 0.01 & 1.28 & 1.10 & $0 .<5$ & 0.050 & .00 & , & 400 \\
\hline $3 R-4,121-123$ & 174.430 & 0.908 & & 0.3 & 0.6 & 2.2 & & & & & & & & \\
\hline 30 . & & & & & 0. & 1. & & & & & & & & \\
\hline $3 \mathrm{R}-5,45-46$ & & 0.9 & 1072 & 0. & 0.4 & 1.7 & 0.24 & 1.15 & 0.90 & 0.20 & 0.040 & 0.33 & 6 & 520 \\
\hline 3R-5, $107-108$ & 175.790 & 0.907 & & 0.2 & 0.6 & 2.2 & & & & & & & & \\
\hline $3 R-6,83-84$ & 177.050 & 0.892 & 1127 & 0.4 & 0.8 & 2.7 & 0.45 & 1.88 & 1.60 & 0.30 & 0.060 & 0.45 & 8 & 670 \\
\hline
\end{tabular}

Note: $1-\sigma$ uncertainties are $1 \%-3 \%$ for $\mathrm{Fe}, \mathrm{Sc}, \mathrm{Th}, \mathrm{Ce}, \mathrm{Cr}, \mathrm{Co}, \mathrm{Eu} ; 3 \%-5 \%$ for $\mathrm{CaCO}_{3}, \mathrm{Si}, \mathrm{Sr}, \mathrm{Cs}, \mathrm{Zn}, \mathrm{Hf}, \mathrm{Tb}, \mathrm{Yb}, \mathrm{La}, \mathrm{Sm} ; 5 \%-10 \%$ for $\mathrm{Ba}, \mathrm{Rb}, \mathrm{Lu}$; and $10 \%-15 \%$ for $\mathrm{Al}, \mathrm{Mg}, \mathrm{Ni}, \mathrm{Nd}$.

$2 \mathrm{R}-2,20-22 \mathrm{~cm}$, and $122-761 \mathrm{C}-2 \mathrm{R}-3,70-72 \mathrm{~cm})$, Core $122-$ $761 \mathrm{C}-2 \mathrm{R}$ is mainly of reversed polarity. Core $122-761 \mathrm{C}-3 \mathrm{R}$ shows the following polarity sequence: reversed at the top, normal from 170.72 to 171.92 mbsf and reversed from 172.25 mbsf to the base of the core at 177.05 mbsf.

Comparison of this polarity pattern with the polarity time scale of Haq et al. (1987), aided by paleontological data (Haq, von Rad, O'Connell, et al., 1990), leads to the identification of Chrons 26 to 29 (Fig. 7). The lower part of Core 122-761C-3R corresponds to the reversed-polarity Chron 29R; the base of this chron was not cored at Hole 761C. The upper boundary of this chron with the normal-polarity Chron $29 \mathrm{~N}$ occurs between Sample 122-761C-3R-3, 20-22 cm (171.92 mbsf), and Sample 122-761C-3R-3, 53-55 cm (172.25 mbsf). The bound- ary between Chron $29 \mathrm{~N}$ and reversed-polarity Chron 28R occurs between Sample 122-761C-3R-1, 63-65 cm (170.33 mbsf), and Sample 122-761C-3R-1, 102-104 cm (170.72 mbsf). In the absence of a base for Core $122-761 C-2 R$, the correlation of the polarity sequence of this core with the polarity reference time scale is less straightforward. However, biozonation shows that it is likely to correspond with the upper part of the reversed-polarity Chron 27R and Chron 26.

\section{Sedimentation Rate}

Sedimentation rates are derived from the positions of the top and base of Chron 29. According to Haq et al. (1988), the top of Chron $29 \mathrm{~N}$ occurs at $64.91 \mathrm{Ma}$ and the base of Chron $29 \mathrm{R}$ at $66.96 \mathrm{Ma}$, the $\mathrm{K} / \mathrm{T}$ boundary itself being at $66.5 \mathrm{Ma}$. 
Table 1 (continued).

\begin{tabular}{|c|c|c|c|c|c|c|c|c|c|c|c|c|c|}
\hline $\begin{array}{c}\mathrm{Zn} \\
\mathrm{ppm}\end{array}$ & $\begin{array}{c}\text { La } \\
\text { pnm }\end{array}$ & $\begin{array}{r}\mathrm{Ce} \\
\mathrm{ppm}\end{array}$ & $\begin{array}{c}\mathrm{Nd} \\
\mathrm{ppm}\end{array}$ & $\begin{array}{l}\mathrm{Sm} \\
\mathrm{ppm}\end{array}$ & $\begin{array}{c}\text { Eu } \\
\text { ppm }\end{array}$ & $\begin{array}{c}\text { To } \\
\text { ppm }\end{array}$ & $\begin{array}{c}\text { Yo } \\
\text { ppm }\end{array}$ & $\begin{array}{r}\text { Lu } \\
\text { ppm }\end{array}$ & $\begin{array}{c}\mathrm{Cr} \\
\mathrm{ppm}\end{array}$ & $\begin{array}{c}\text { Co } \\
\text { ppm }\end{array}$ & $\begin{array}{c}\mathrm{Ni} \\
\text { ppm }\end{array}$ & & $\begin{array}{l}\text { Ir } \\
\text { ppb }\end{array}$ \\
\hline \multirow[t]{3}{*}{$\begin{array}{l}19 \\
35\end{array}$} & $\begin{array}{r}9.0 \\
12.5\end{array}$ & $\begin{array}{r}8.0 \\
13.9\end{array}$ & $\begin{array}{r}7.0 \\
10.7\end{array}$ & $\begin{array}{l}1.90 \\
2.60\end{array}$ & $\begin{array}{l}0.32 \\
0.46\end{array}$ & $\begin{array}{l}0.22 \\
0.33\end{array}$ & $\begin{array}{l}0.75 \\
1.00\end{array}$ & $\begin{array}{l}0.12 \\
0.16\end{array}$ & $\begin{array}{l}11.0 \\
25.0\end{array}$ & $\begin{array}{l}1.58 \\
3.15\end{array}$ & 12.0 & & \\
\hline & & & & & & & & & & & & 0.022 & \pm 0.008 \\
\hline & & & & & & & & & & & & $\begin{array}{l}0.022 \\
0.032 \\
0.061 \\
0.038\end{array}$ & $\begin{array}{l} \pm 0.009 \\
\pm 0.026 \\
\pm 0.022 \\
\pm 0.025\end{array}$ \\
\hline 64 & 24.0 & 31.0 & 18.0 & 4.90 & 0.85 & 0.70 & 2.20 & 0.34 & 54.0 & 5.20 & 23.0 & & \\
\hline 69 & 25.0 & 33.0 & 19.4 & 5.20 & 0.85 & 0.70 & 2.20 & 0.41 & 57.0 & 5.64 & 22.0 & 0.058 & \pm 0.012 \\
\hline 60 & 24.0 & 31.0 & 17.0 & 5.20 & 0.80 & 0.60 & 2.10 & 0.42 & 56.0 & 5.10 & & 0.049 & \pm 0.017 \\
\hline 47 & 20.0 & 23.4 & 16.0 & 4.50 & 0.66 & 0.50 & 1.85 & 0.29 & 39.0 & 4.35 & 17.0 & 0.063 & \pm 0.011 \\
\hline 38 & 13.0 & 18.0 & 13.2 & 2.90 & 0.59 & 0.42 & 1.40 & 0.24 & 30.0 & 3.69 & & 0.053 & \pm 0.020 \\
\hline 51 & 22.0 & 22.0 & 15.0 & 5.50 & 0.77 & 0.57 & 1.80 & 0.31 & 38.0 & 4.50 & & 0.051 & \pm 0.018 \\
\hline 48 & 18.0 & 20.4 & 14.0 & 4.30 & 0.67 & 0.50 & 1.50 & 0.27 & 36.0 & 4.20 & & 0.058 & \pm 0.035 \\
\hline 48 & 17.0 & 20.0 & 14.0 & 3.90 & 0.67 & 0.50 & 1.50 & 0.24 & 35.0 & 4.30 & & 0.047 & \pm 0.020 \\
\hline 42 & 16.0 & 18.0 & 13.0 & 3.60 & 0.60 & 0.48 & 1.46 & 0.24 & 30.0 & 3.70 & & 0.052 & \pm 0.016 \\
\hline 44 & 19.0 & 22.0 & 16.7 & 4.50 & 0.70 & 0.60 & 1.80 & 0.26 & 37.0 & 5.20 & & 0.091 & \pm 0.035 \\
\hline 54 & 20.0 & 22.0 & 14.0 & 4.60 & 0.74 & 0.55 & 1.70 & 0.30 & 42.0 & 5.47 & & 0.120 & \pm 0.020 \\
\hline 54 & 23.0 & 22.0 & 14.0 & 4.40 & 0.73 & 0.60 & 2.00 & 0.34 & 37.0 & 4.85 & 25.0 & 0.171 & \pm 0.024 \\
\hline 62 & 23.0 & 24.0 & 17.0 & 4.30 & 0.76 & 0.60 & 2.00 & & 38.0 & 5.00 & & 0.244 & \pm 0.026 \\
\hline 58 & 24.0 & 23.0 & 16.0 & 4.50 & 0.73 & 0.60 & 2.00 & 0.33 & 40.0 & 4.85 & 20.0 & 0.094 & \pm 0.033 \\
\hline 57 & 22.0 & 23.0 & 16.0 & 4.50 & 0.71 & 0.57 & 1.90 & 0.29 & 37.0 & 5.20 & 30.0 & 0.156 & \pm 0.007 \\
\hline 65 & 23.0 & 22.0 & 15.0 & 4.20 & 0.70 & 0.60 & 2.10 & 0.28 & 36.0 & 4.60 & 20.0 & 0.171 & \pm 0.014 \\
\hline 61 & 24.0 & 23.0 & 17.0 & 4.60 & 0.74 & 0.57 & 2.00 & 0.35 & 37.0 & 4.80 & 22.0 & 0.139 & \pm 0.014 \\
\hline 55 & 22.0 & 22.0 & 15.3 & 4.20 & 0.67 & 0.60 & 1.90 & 0.32 & 36.0 & 4.60 & 20.0 & 0.156 & \pm 0.022 \\
\hline 57 & 24.0 & 21.4 & 17.0 & 4.60 & 0.70 & 0.55 & 2.00 & 0.35 & 35.0 & 4.40 & 10.0 & 0.234 & \pm 0.015 \\
\hline 56 & 25.0 & 22.0 & 18.2 & 4.70 & 0.72 & 0.55 & 1.95 & 0.32 & 38.0 & 4.75 & & 0.297 & \pm 0.021 \\
\hline 67 & 27.0 & 28.0 & 20.6 & 5.20 & 0.92 & 0.72 & 2.20 & 0.35 & 49.0 & 5.90 & 21.0 & 0.342 & \pm 0.041 \\
\hline 62 & 25.0 & 24.5 & 17.8 & 4.60 & 0.82 & 0.70 & 2.20 & 0.33 & 40.0 & 4.70 & 23.0 & 0.363 & \pm 0.023 \\
\hline 79 & 29.0 & 30.0 & 20.5 & 5.70 & 1.00 & 0.80 & 2.30 & 0.34 & 52.0 & 5.85 & & 0.405 & \pm 0.027 \\
\hline 56 & 32.0 & 25.6 & 23.0 & 6.10 & 0.88 & 0.70 & 2.20 & 0.37 & 44.0 & 4.20 & 3.0 & 0.518 & \pm 0.029 \\
\hline 38 & 20.0 & 16.0 & 15.4 & 4.30 & 0.60 & 0.47 & 1.38 & 0.26 & 31.0 & 2.80 & 28.0 & 2.153 & \pm 0.042 \\
\hline 57 & 21.0 & 21.5 & 20.0 & 4.70 & 0.80 & 0.60 & 1.60 & 0.26 & 44.0 & 3.80 & 21.0 & 2.289 & \pm 0.055 \\
\hline 38 & & 15.5 & 13.0 & & 0.69 & 0.46 & 1.15 & & 45.0 & 3.20 & & 4.543 & \pm 0.076 \\
\hline 38 & 18.0 & 14.0 & 14.6 & 3.70 & 0.61 & 0.41 & 1.20 & 0.18 & 60.0 & 3.60 & 12.0 & 4.777 & \pm 0.133 \\
\hline 39 & 18.0 & 14.0 & 14.3 & 3.62 & 0.59 & 0.41 & 1.25 & 0.18 & 53.5 & 3.07 & 22.7 & 5.333 & \pm 0.250 \\
\hline 57 & 16.3 & 11.4 & 12.5 & 2.93 & 0.48 & 0.32 & 1.05 & 0.17 & 50.4 & 2.81 & 15.0 & 4.990 & \pm 0.240 \\
\hline 32 & 16.1 & 12.5 & 12.9 & 3.05 & 0.51 & 0.37 & 1.13 & 0.17 & 65.3 & 3.28 & 23.2 & 5.912 & \pm 0.220 \\
\hline 29 & 14.3 & 11.4 & 10.8 & 2.54 & 0.45 & 0.31 & 1.01 & 0.15 & 76.0 & 3.11 & 25.2 & 7.356 & \pm 0.270 \\
\hline 26 & 13.4 & 10.5 & 12.8 & 2.34 & 0.41 & 0.28 & 1.02 & 0.16 & 76.4 & 2.96 & 25.8 & 7.490 & \pm 0.520 \\
\hline 50 & 13.3 & 9.6 & 9.1 & 2.51 & 0.40 & 0.29 & 0.94 & 0.15 & 79.3 & 3.05 & 27.6 & 7.680 & \pm 0.240 \\
\hline 28 & 12.7 & 9.4 & 8.2 & 2.15 & 0.37 & 0.27 & 0.92 & 0.15 & 86.6 & 2.97 & 27.9 & 7.663 & \pm 0.250 \\
\hline 25 & 12.5 & 9.6 & 8.4 & 2.15 & 0.38 & 0.28 & 0.97 & 0.15 & 83.3 & 2.98 & 31.3 & 7.622 & \pm 0.310 \\
\hline 14 & 10.9 & 6.3 & 6.1 & 1.58 & 0.26 & 0.20 & 0.92 & 0.15 & 30.5 & 1.54 & 10.9 & 2.866 & \pm 0.130 \\
\hline 16 & 9.5 & 6.4 & 5.9 & 1.45 & 0.24 & 0.20 & 0.95 & 0.14 & 13.5 & 1.32 & 7.1 & 2.212 & \pm 0.150 \\
\hline 15 & 9.8 & 6.2 & 5.9 & 1.44 & 0.25 & 0.20 & 0.90 & 0.14 & 10.3 & 1.35 & 5.5 & 1.810 & \pm 0.150 \\
\hline 12 & 8.0 & 6.5 & 5.0 & 1.40 & 0.22 & 0.18 & 0.70 & 0.13 & 6.8 & 1.20 & & 0.235 & \pm 0.018 \\
\hline 18 & 8.0 & 6.7 & 5.0 & 1.40 & 0.24 & 0.19 & 0.80 & 0.11 & 5.3 & 1.30 & & 0.141 & \pm 0.009 \\
\hline 16 & 9.0 & 6.7 & 5.5 & 1.50 & 0.24 & 0.20 & 0.70 & 0.14 & 5.3 & 1.30 & & 0.156 & \pm 0.010 \\
\hline 14 & 8.0 & A 6 & 5.0 & 1.50 & 0.23 & 0.19 & 0.70 & 0.12 & 5.2 & 1.20 & & 0.109 & \pm 0.009 \\
\hline 17 & 5.u & 5.0 & 6.1 & 1.40 & 0.24 & 0.20 & 0.70 & 0.11 & 5.2 & 1.11 & & 0.091 & \pm 0.008 \\
\hline 17 & 7.0 & $\dot{j u}$ & \$.O & 1.90 & 0.25 & 0.20 & 0.70 & 0.09 & 7.0 & 1.20 & & 0.077 & \pm 0.007 \\
\hline 12 & & 5.0 & 5.0 & & 0.20 & 0.14 & 0.50 & & 4.3 & 0.91 & & 0.071 & \pm 0.004 \\
\hline 16 & & 6.2 & 5.9 & & 0.25 & 0.17 & 0.63 & & 5.3 & 1.08 & & 0.058 & \pm 0.003 \\
\hline & & & & & & & & & & & & 0.066 & \pm 0.014 \\
\hline & & & & & & & & & & & & 0.070 & \pm 0.017 \\
\hline 14 & 8.0 & 6.4 & 5.0 & 1.40 & 0.23 & 0.18 & 0.65 & 0.10 & 5,1 & 1.02 & & 0.014 & \pm 0.002 \\
\hline & & & & & & & & & & & & 0.005 & \pm 0.003 \\
\hline 12 & 8.0 & 6.2 & 4.0 & 1.50 & 0.24 & 0.21 & 0.70 & 0.13 & 4.2 & 1.15 & & & \\
\hline & & & & & & & & & & & & 0.004 & \pm 0.003 \\
\hline 21 & 12.2 & 10.0 & 7.9 & 2.20 & 0.38 & 0.30 & 1.00 & 0.16 & 6.1 & 1.81 & & 0.005 & \pm 0.002 \\
\hline
\end{tabular}

The Maestrichtian part of Chron 29 (about $46010^{3} \mathrm{yr}$ ) extends over at least $4.58 \mathrm{~m}$. The thickness of the Danian part of Chron 29 (about $1.5910^{6} \mathrm{yr}$ ) is between $1.75 \mathrm{~m}$ and $2.14 \mathrm{~m}$. The average sedimentation rate is therefore higher than 10.4 $\mathrm{m} / \mathrm{m} . \mathrm{y}$. in the Maestrichtian and between 1.1 and $1.3 \mathrm{~m} / \mathrm{m} . \mathrm{y}$., at the base of the Danian, nearly 10 times lower. The data showing the carbonate and insoluble fraction deposition rates are displayed in Table 3 .

Data from Core $122-761 C-2 \mathrm{R}$ permit the evaluation of the sedimentation rate on a longer time scale. The top of Chron 27 (61.36 Ma) is around $164 \mathrm{mbsf}$, about $8-9 \mathrm{~m}$ above the boundary. The average sedimentation rate during most of the Danian can therefore be estimated at about $1.7 \mathrm{~m} / \mathrm{m}$.y., close to the value found for the basal Danian. This shows that the rate of deposition was rather low and stable for the few millions years following the $\mathrm{K} / \mathrm{T}$ event, in agreement with the high and nearly constant insoluble fraction observed during that period.

\section{DISCUSSION}

The onset of the main Ir anomaly is very sharp and occurs in Sample 122-761C-3R-3, 75-76 cm, in a part of the core which seems to be free of coring disturbance (see core picture in Haq, von Rad, O'Connell, et al., 1990). The presence of siliceous nodules around Sections $122-761 \mathrm{C}-3 \mathrm{R}-3,65 \mathrm{~cm}$, and $122-761 \mathrm{C}-3 \mathrm{R}-3,80 \mathrm{~cm}$, could have locally damaged the quality of the core but has not produced any visible feature in the $\mathrm{Ir}$ distribution. The difference in position between the Ir-defined 


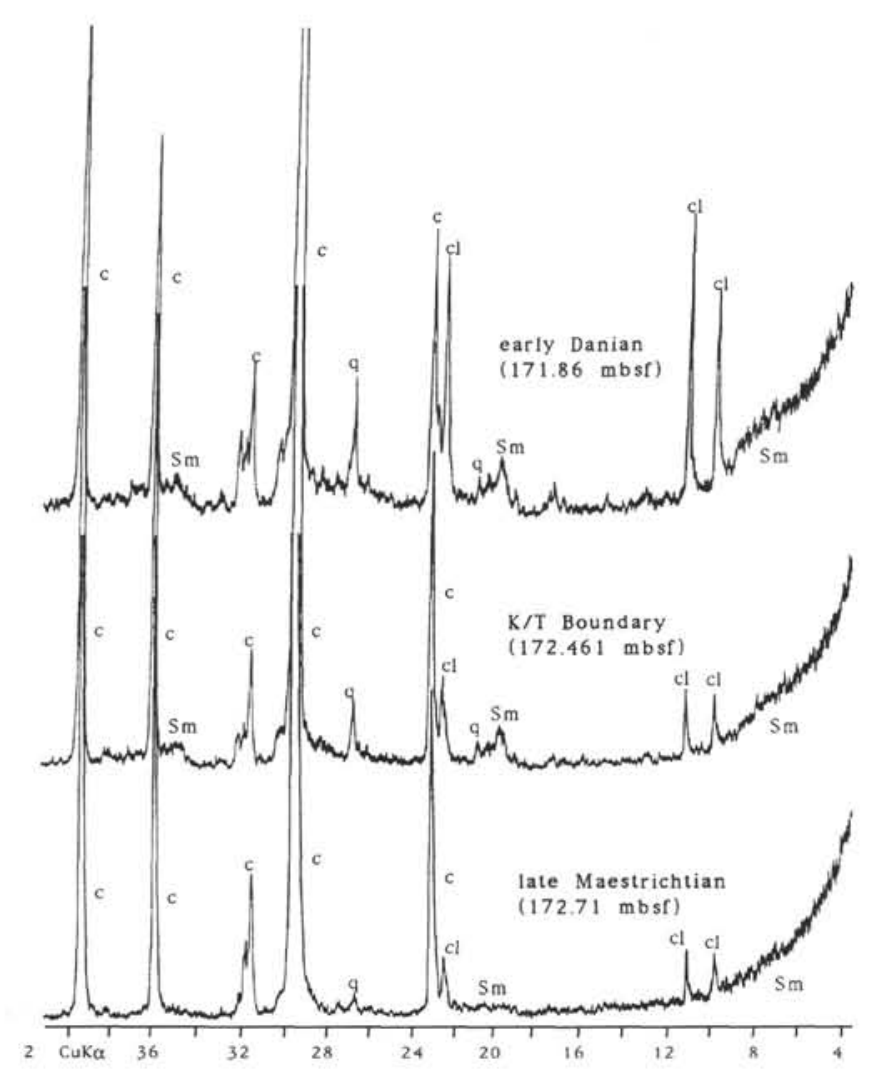

Figure 4. Diffraction spectra of whole-rock samples showing calcite (c), clinoptilolite (cl), quartz (q), and smectite (Sm) lines. No change of the mineralogical components occurs across the boundary.

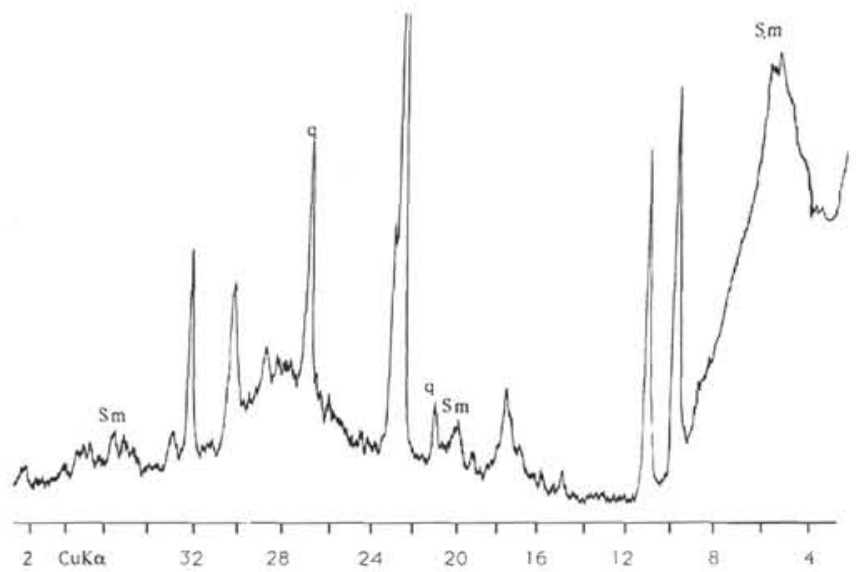

Figure 5. Typical diffraction spectrum of a decarbonated sample. All samples display similar spectrum showing well-crystallized phases. Only smectite (Sm) and quartz (q) lines are noted; others are those of clinoptilolite.

boundary (Sample 122-761C-3R-3, 75-76 cm, $172.46 \mathrm{mbsf}$ ) and the biostratigraphic position (Sample 122-761C-3R-3, $67-68 \mathrm{~cm}, 172.38 \mathrm{mbsf}$; the base of Zone NP1) is essentially a matter of definition. As pointed out by Smit and Romein (1985), in all complete sections the nannofossil-defined $\mathrm{K} / \mathrm{T}$ boundary (first occurrence of Tertiary nannofossils) is located above the mass extinction level which is systematically observed in coincidence with the rapid increase of the Ir con- centration. So, the boundary, if defined by the mass extinction level or the Ir distribution, should be moved down by $8-9 \mathrm{~cm}$ with respect to the previously quoted position based on the first occurrence of Tertiary nannofossils (Haq, von Rad, O'Connell, et al., 1990).

The meaning of the low Ir excess that extends symmetrically on both sides of the main anomaly is not clear. Officer and Drake (1985) showed that it cannot be explained by the bioturbation of a quasi-instantaneous event. Does it mean that the Ir distribution represents a long duration event? Two types of scenarios involving multiple episodes have been proposed to explain the $\mathrm{K} / \mathrm{T}$ boundary features. Hut et al. (1987) propose that multiple collisions of comets explain the stepwise precursory biological extinctions (Keller, 1989) occurring at the end of the Maestrichtian. Officer and Drake (1983) and Courtillot and Cisowski (1987) postulate the existence of a period of intense volcanic activity associated with the Deccan traps formation. In this paper, without entering into the debate, we can, however, note that the Ir distribution curve is rather smooth and does not indicate the occurrence of multiple discrete events. Comparison with other $\mathrm{K} / \mathrm{T}$ sections provide useful information. At Hole 761C, the Ir anomaly, shoulder included, covers an interval of about $10^{6}$ years, suggesting an event of long duration. However, in several European sections the Ir excess is also observed over about 1-2 m, which represents only $10^{4}-10^{5} \mathrm{yr}$ of deposition (Rocchia et al. 1987). This apparent inconsistency suggests that the full extent of the Ir anomaly does not represent the actual duration of the $\mathrm{K} / \mathrm{T}$ event. It is more likely due to postdepositional geochemical processes.

The magnetostratigraphic study of Cores $122-761 \mathrm{C}-2 \mathrm{R}$ and $122-761 C-3 R$ has permitted the identification of the magnetic polarity sequence near the $\mathrm{K} / \mathrm{T}$ boundary and a reasonably accurate estimate of the sedimentation rates in the late Maestrichtian and early Danian. The planktonic crisis reduced the carbonate deposition rate by a factor of 10 . The sedimentation rate of the insoluble fraction also suffered a smaller but still important reduction by a factor of 3 to 4 . This is a surprising but not new fact; it has already been observed in many $\mathrm{K} / \mathrm{T}$ sections (Zachos et al., 1985; Rocchia et al., 1987; Timothy and D'Hondt, 1990). This reduction of the insoluble-fraction deposition rate is not a local feature but appears to be global. Until now, it has not been convincingly explained. Indeed, it is easy to understand that the planktonic turnover at the boundary could significantly change the carbonate production. That it could also reduce the deposition rate of the nonbiogenic component is not obvious at all. It implies that the machinery of sediment production and transportation has been disturbed on a global scale for millions of years.

With the deposition rates derived from magnetostratigraphy, we can check the consistency of our Ir background estimates with the value of the steady-state Ir infall. As pointed out above, our measurements are not really statistically significant and they permit us only to derive upper limits. With a 3- $\sigma$ criterion we can say that Ir concentrations are lower than $10 \mathrm{pg} / \mathrm{g}$ for the Maestrichtian and $45 \mathrm{pg} / \mathrm{g}$ for the Danian. According to Kyte and Wasson (1986), the steady-state extraterrestrial Ir flux is 9 $\mathrm{ng} / \mathrm{cm}^{2} / \mathrm{m}$.y. We have not included in that budget the terrestrial contribution, which can change from one site to the other. Assuming constant sedimentation rates below $(>10 \mathrm{~m} / \mathrm{m} . \mathrm{y}$.) and above the $\mathrm{K} / \mathrm{T}$ boundary $(1.6 \mathrm{~m} / \mathrm{m}$.y. between the $\mathrm{K} / \mathrm{T}$ boundary and top of Chron 27), the Ir concentration should be lower than $6 \mathrm{pg} / \mathrm{g}$ during the Maestrichtian part of Chron 29R and about 45 $\mathrm{pg} / \mathrm{g}$ up to the top of Core $122-761 \mathrm{C}-2 \mathrm{R}$. Taking into account the large uncertainties of our measurements and of the Kyte and Wasson budget, we can conclude that they are consistent with each other for the Maestrichtian and for the Danian as well. It is 


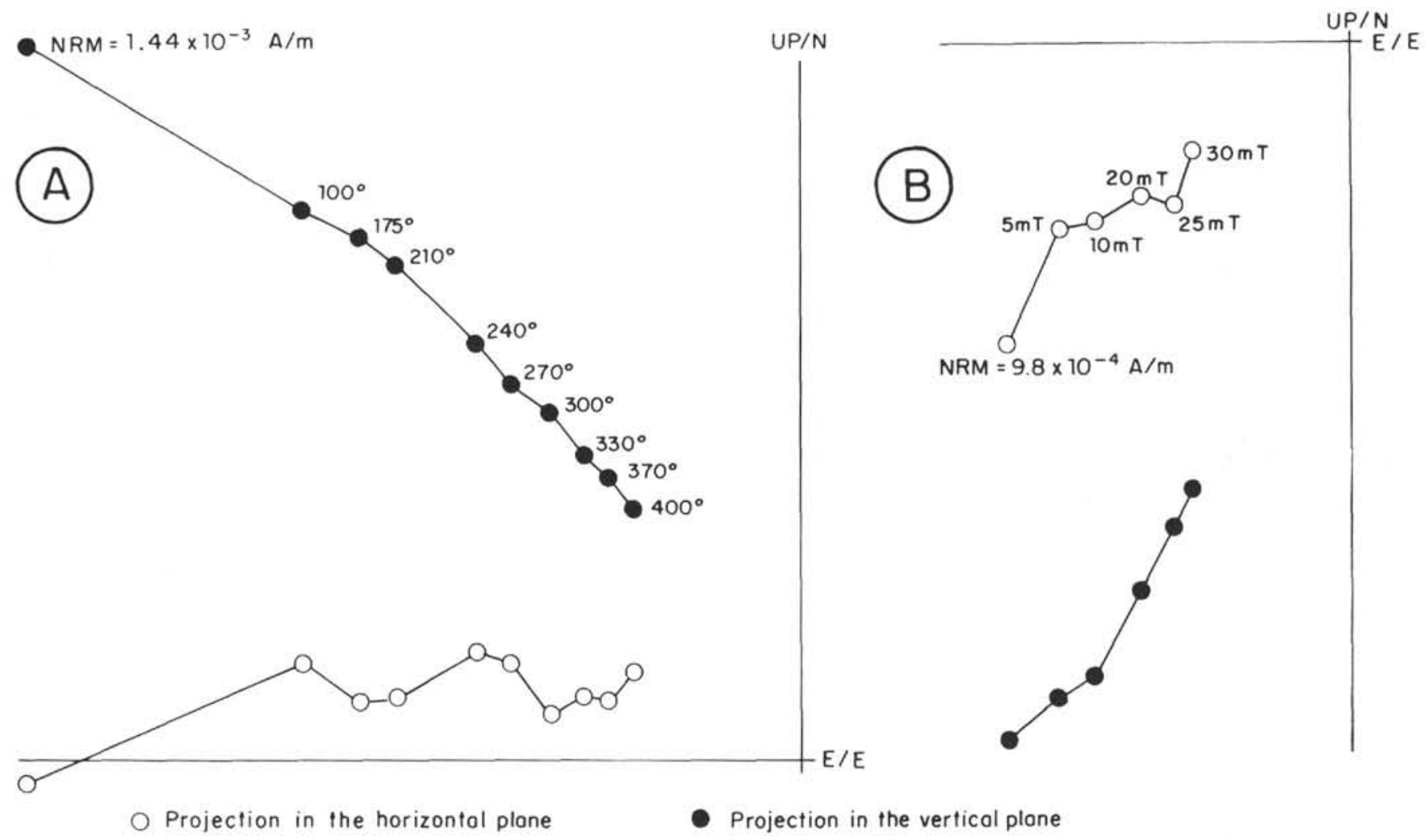

Figure 6. Orthogonal vector plots of demagnetization curves for two samples illustrating typical magnetic behaviors. A. Thermal demagnetization, Sample 122-761C-2R-2, 20-22 cm. B. AF demagnetization, Sample 122-761C-2R-2, 124-126 cm. An initial unstable component is removed by $100^{\circ} \mathrm{C}$ heating or by $5-10 \mathrm{mT}$ AF demagnetization. Above those values the ChRM directions are defined and are of normal (A) or reversed (B) polarity.

Table 2. Direction of characteristic magnetization, $\mathrm{K} / \mathrm{T}$ boundary (Cores 122-761C-2R and -3R).

\begin{tabular}{lllcc}
\hline $\begin{array}{c}\text { Core, section, } \\
\text { interval (cm) }\end{array}$ & $\begin{array}{c}\text { Depth } \\
(\mathrm{mbsf})\end{array}$ & $\begin{array}{c}\text { NRM } \\
(\mathrm{A} / \mathrm{m})\end{array}$ & $\begin{array}{c}\text { Inclination } \\
\left({ }^{\circ}\right)\end{array}$ & $\begin{array}{c}\text { Demagnetization } \\
\text { step }\end{array}$ \\
\hline 122-761C- & & & & \\
2R-1, 102 & 161.22 & $3.91 \mathrm{E}-3$ & +24 & $30 \mathrm{mT}$ \\
2R-2, 20 & 161.90 & $1.44 \mathrm{E}-3$ & -51 & $400^{\circ} \mathrm{C}$ \\
2R-2, 71 & 162.41 & $7.50 \mathrm{E}-5$ & +70 & $370^{\circ} \mathrm{C}$ \\
2R-2, 124 & 162.94 & $9.82 \mathrm{E}-4$ & +50 & $30 \mathrm{mT}$ \\
2R-3, 20 & 163.40 & $8.10 \mathrm{E}-5$ & +57 & $400^{\circ} \mathrm{C}$ \\
2R-3, 70 & 163.90 & $2.19 \mathrm{E}-4$ & -50 & $400^{\circ} \mathrm{C}$ \\
2R-3, 118 & 164.38 & $2.80 \mathrm{E}-5$ & +69 & $30 \mathrm{mT}$ \\
2R-3, 140 & 164.60 & $5.50 \mathrm{E}-5$ & +54 & $400^{\circ} \mathrm{C}$ \\
2R-4, 51 & 165.21 & $4.10 \mathrm{E}-5$ & +72 & $30 \mathrm{mT}$ \\
3R-1, 48 & 170.18 & $5.60 \mathrm{E}-5$ & +54 & $400^{\circ} \mathrm{C}$ \\
3R-1, 63 & 170.33 & $4.70 \mathrm{E}-5$ & +60 & $30 \mathrm{mT}$ \\
3R-1, 102 & 170.72 & $5.00 \mathrm{E}-5$ & -44 & $300^{\circ} \mathrm{C}$ \\
3R-1, 140 & 171.10 & $1.09 \mathrm{E}-4$ & -50 & $370^{\circ} \mathrm{C}$ \\
3R-2, 14 & 171.34 & $6.50 \mathrm{E}-5$ & -26 & $330^{\circ} \mathrm{C}$ \\
3R-2, 48 & 171.68 & $1.15 \mathrm{E}-4$ & -37 & $30 \mathrm{mT}$ \\
3R-3, 20 & 171.92 & $1.01 \mathrm{E}-4$ & -48 & $300^{\circ} \mathrm{C}$ \\
3R-3, 53 & 172.25 & $9.70 \mathrm{E}-5$ & +45 & $270^{\circ} \mathrm{C}$ \\
3R-3, 112 & 172.84 & $1.08 \mathrm{E}-4$ & +24 & $30 \mathrm{mT}$ \\
3R-4, 21 & 173.43 & $1.70 \mathrm{E}-4$ & +29 & $400^{\circ} \mathrm{C}$ \\
3R-4, 59 & 173.81 & $8.00 \mathrm{E}-5$ & +50 & $30 \mathrm{mT}$ \\
3R-4, 121 & 174.43 & $1.02 \mathrm{E}-4$ & +55 & $400^{\circ} \mathrm{C}$ \\
3R-5, 45 & 175.17 & $5.30 \mathrm{E}-5$ & +62 & $30 \mathrm{mT}$ \\
3R-5, 107 & 175.79 & $1.95 \mathrm{E}-4$ & +53 & $400^{\circ} \mathrm{C}$ \\
3R-6, 18 & 176.40 & $8.30 \mathrm{E}-5$ & +62 & $30 \mathrm{mT}$ \\
3R-6, 83 & 177.05 & $9.10 \mathrm{E}-5$ & +67 & $400^{\circ} \mathrm{C}$ \\
\hline & & & & \\
\hline
\end{tabular}

important to note that the Danian background was measured in samples that are rather close to the upward-extending part of the Ir distribution. A different estimate could be obtained in more distant samples. Additional measurements up to the top of Core $122-761 \mathrm{C}-2 \mathrm{R}$ are required to corroborate that point.

As far as the nature of the $\mathrm{K} / \mathrm{T}$ event is concerned, all observations point to a cosmic origin. The presence of $\mathrm{Ir}$ and its ratio in chondritic proportion with $\mathrm{Cr}$ and $\mathrm{Fe}$ strongly support a dramatic increase of the cosmic matter accretion rate at the $\mathrm{K} / \mathrm{T}$ boundary. The significantly lower Ni enrichment is not inconsistent with this hypothesis. It is a common feature already observed in other $\mathrm{K} / \mathrm{T}$ sites and could be due to dissolution of $\mathrm{Ni}$ oxide in seawater (Alvarez et al. 1980). The presence of Ni-rich magnetites often clustered in flattened spheroids provides the most convincing signature for an extraterrestrial origin. These minerals have no counterparts in terrestrial materials (Smit and Kyte, 1984; Kyte and Smit, 1986). Smaller crystals of this type are abundant in micrometeorites (Brownlee, 1981; Bonté et al., 1987; Robin et al., 1990) but, with their composition and size, magnetites from Hole $761 \mathrm{C}$ look more like those found in meteoritic ablation products from a Jurassic sequence from northern Italy (Jéhanno et al., 1987). In this Jurassic site, Ni-rich magnetites are locked in host bodies which, because of their perfectly preserved internal structures, can be unambiguously identified with cosmic particulates. Conversely, magnetites of the $\mathrm{K} / \mathrm{T}$ boundary at Hole 761C are located in highly weathered, altered, and damaged bodies that cannot be immediately identified on purely morphological considerations. However, their chemical composition shows that they are likely to constitute the relics of pure meteoritic debris. 


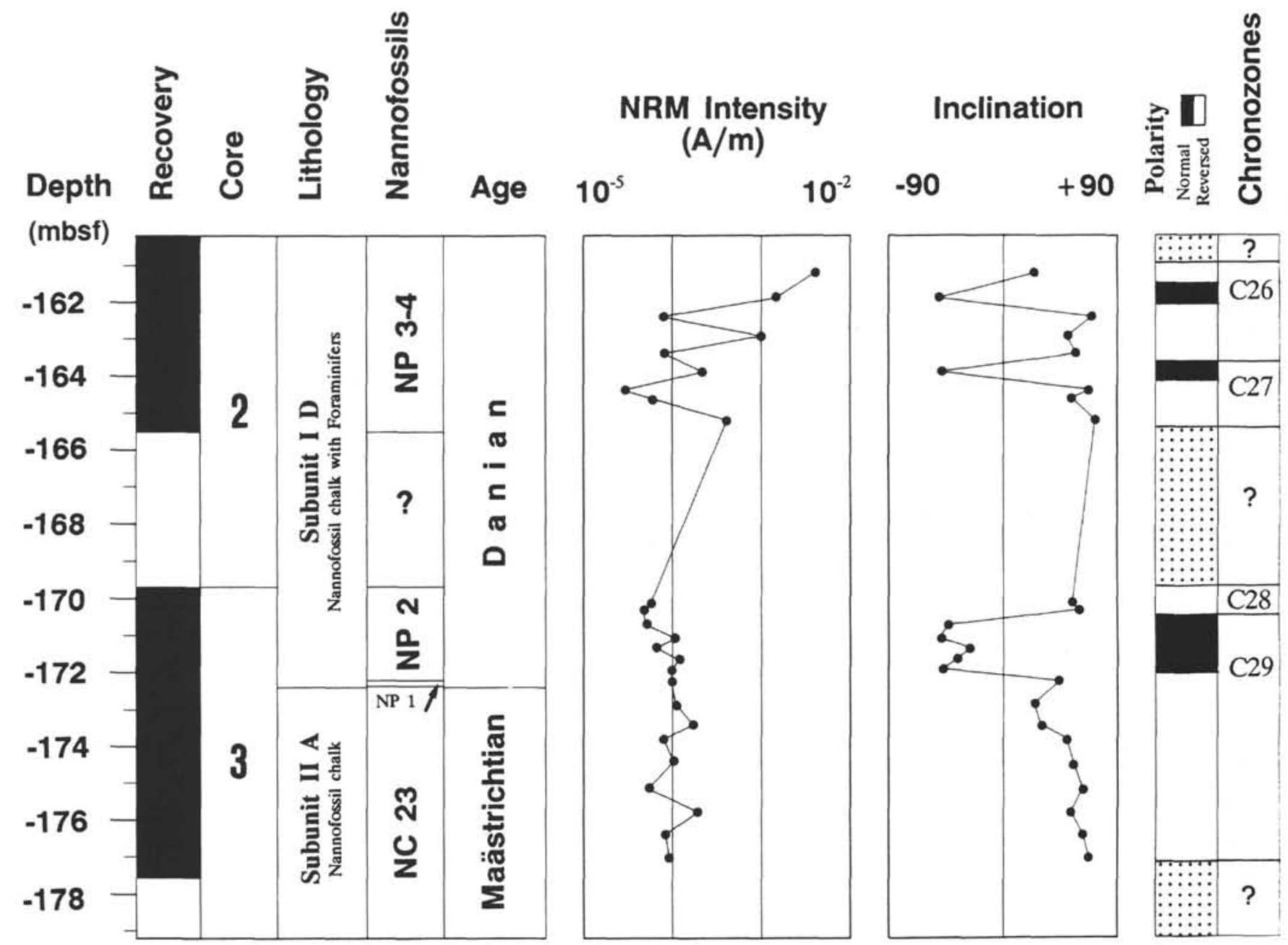

Figure 7. Maestrichtian/Danian boundary magnetostratigraphy of Hole 761C.

Table 3. Magnetostratigraphy and sedimentation parameters.

\begin{tabular}{|c|c|c|c|}
\hline \multirow[b]{2}{*}{ Position (mbsf) } & \multicolumn{3}{|c|}{ Extent of Chron 29} \\
\hline & $\begin{array}{c}\text { Bottom of } \\
\text { Chron } 29 \mathrm{R} \\
>177.05\end{array}$ & $\begin{array}{c}\text { K/T Boundary } \\
172.47\end{array}$ & $\begin{array}{c}\text { Top of Chron } 29 \mathrm{~N} \\
170.33-170.72\end{array}$ \\
\hline Sedimentation parameters & & Maestrichtian & Danian \\
\hline Thickness of Chron $29(\mathrm{~m})$ & & $>4.58$ & 1.75 to 2.14 \\
\hline Sedimentation rate $(\mathrm{m} / \mathrm{m} . \mathrm{y})$. & & $>10.4$ & 1.1 to 1.3 \\
\hline Insoluble fraction (\%) & & $\approx 9$ & $\approx 30$ \\
\hline Density $\left(\mathrm{g} / \mathrm{cm}^{3}\right)$ & & 1.45 & 1.3 \\
\hline $\begin{array}{l}\text { Mass accumulation rate } \\
\left(\mathrm{g} / \mathrm{cm}^{2} / 1000 \mathrm{yr}\right)\end{array}$ & & $>1.51$ & 0.14 to 0.17 \\
\hline $\begin{array}{l}\text { Carbonate accumulation rate } \\
\left(\mathrm{g} / \mathrm{cm}^{2} / 1000 \mathrm{yr}\right)\end{array}$ & & $>1.37$ & 0.1 to 0.12 \\
\hline $\begin{array}{l}\text { Insoluble fraction } \\
\text { accumulation rate } \\
\left(\mathrm{g} / \mathrm{cm}^{2} / 1000 \mathrm{yr}\right)\end{array}$ & & $>0.14$ & 0.043 to 0.051 \\
\hline
\end{tabular}

Can we conclude that all the observations at Hole 761C support the hypothesis of a comet or asteroid impact? Such events should have produced a considerable amount of ejecta, 10 to 100 times the mass of the impactor according to collisional models (O'Keefe and Ahrens, 1982). In Hole 761C this ejecta does not appear. The lack of a boundary clay layer, the constant mineralogical composition across the boundary, and the absence of chemical anomalies other than those of meteoritic elements suggest that the amount of ejecta is likely lower than one impactor mass. The slight change of the $\mathrm{La} / \mathrm{Yb}$ ratio is probably not produced by an exotic component because the maximum occurs above the $\mathrm{K} / \mathrm{T}$ boundary. It might be related to the drastic reduction of the carbonate productivity.

In short, it seems that the $0.16 \mathrm{~g} / \mathrm{cm}^{2}$ of $\mathrm{Cl}$-like chondritic material derived from the $\mathrm{Ir}$ flux at Hole $761 \mathrm{C}$ were accreted in a pure state without mixing with an exotic terrestrial component. This observation is not unique; Jin and Schmitt (1989), using elemental results they obtained at Holes 577 and 577B (DSDP Leg 86) and by Michel et al. (1985) at Hole 577B in the central Pacific, estimated that the ejecta was less than half the impactor mass. The study of European sections leads to a similar conclusion (Rocchia et al., 1990). This can be explained in several ways: (1) a single impact did occur, but unlike extraterrestrial material, the ejecta was not evenly distributed over the Earth; or, more likely, (2) a significant fraction of the cosmic material was captured by the Earth as multiple small-sized fragments (fragmented comet, as already suggested by Kyte and Wasson (1982) and Schmitt (1989)), dispersing most of their mass in the atmosphere. Only the larger fragments would have produced craters and local deposits of ejecta, including shocked quartz. Apparently, $\mathrm{K} / \mathrm{T}$ sediments at Holes $761 \mathrm{C}$ and 577 were not deposited close to a crater site. 


\section{CONCLUSION}

Data from the $\mathrm{K} / \mathrm{T}$ boundary at Hole $761 \mathrm{C}$ confirm the worldwide character of the Ir anomaly. The continuity of Ir distribution and the value of the Ir integrated flux $\left(80 \mathrm{ng} / \mathrm{cm}^{2}\right.$, comparable with the $\mathrm{K} / \mathrm{T}$ global average) show that the section is probably complete. In addition to Ir, enrichments in meteoritic elements $(\mathrm{Cr}, \mathrm{Fe}, \mathrm{Co}, \mathrm{Ni})$ are observed in close coincidence with a reduction of the sedimentation rate. Abundant Ni-rich magnetites of cosmic origin are also present in the basal Danian. This collection of data indicates a cosmic origin of the boundary event which, according to the Ir flux, resulted in the accretion of $0.16 \mathrm{~g} / \mathrm{cm}^{2}$ of $\mathrm{Cl}$-like chondritic material. However, the accretion process is unclear. The extent of the Ir anomaly is not necessarily due to a long-duration event. On the other hand, the absence of any identifiable ejecta is not easily explained by a single impact. Other scenarios involving multiple small bodies losing and dispersing most of their mass in the atmosphere should be considered.

\section{ACKNOWLEDGMENTS}

The authors would like to thank the Ocean Drilling Program staff for preparing an exceptional set of samples. They are grateful to C. Pillmore and R. A. Schmitt for reviewing and improving this manuscript. This is CFR contribution no. 1117 and CNRS INSU-DBT contribution no. 224.

\section{REFERENCES}

Alvarez, L. W., Alvarez, W., Asaro, F., and Michel, H. V., 1980. Extraterrestrial cause for the Cretaceous-Tertiary extinction. Science, 208:1095-1108.

Bonté, P., Jéhanno, C., Maurette, M., Brownlee, D. E., 1987. Platinum metals and microstructure in magnetic deep sea cosmic spherules. Proc. Lunar Planet. Sci. Conf. 17th, J. Geophys. Res., 92:E641-E648.

Brownlee, D. E., 1981. Extraterrestrial components in deep sea sediments. In Emiliani C. (Ed.), The Sea: New York (Wiley), $733-762$.

Courtillot, V., and Cisowski, S., 1987. The Cretaceous-Tertiary boundary events: external or internal causes? Eos, 68:193-200.

Haq, B. U., Hardenbol, J., and Vail, P. R., 1987. Chronology of fluctuating sea levels since the Triassic. Science, 235:1156-1167. 1988. Mesozoic and Cenozoic chronostratigraphy and cycles of sea-level change. In Wilgus, C., et al., (Eds.), Sea-Level Change-An Integrated Approach. Soc. Econ. Paleontol. Mineral. Spec. Publ., 42:71-108.

Hut P., Alvarez, W., Elder, W. P., Hansen, T., Kauffman, E. G., Keller, G., Shoemaker, E. M., and Weissman P. R., 1987. Comet showers as a cause of mass extinctions. Nature, 329:118-126.

Jéhanno, C., Boclet, D., Bonté, P., Castellarin, A., Rocchia, R., 1987. Identification of two populations of extraterrestrial particles in a Jurassic hardground of the Southern Alps. Proc. 18th Lunar Planet Sci. Conf., 623-630.

Jin, G.-Y., and Schmitt, R. A., 1989a. Pristine Shatsky Rise K/T marine carbonates yield $\mathrm{Fe}_{\mathrm{NET}} / \mathrm{Ir}_{\mathrm{NET}}=\mathrm{Cl}$ chondritic ratio: no evidence for $\mathrm{Fe}$ from terrestrial crater ejecta rules out direct asteroid-comet impact. I. Observations. Lunar Planet. Sci. Conf. 20th, 20:460-465. (Abstract)

Keller, G., 1989. Extended K/T boundary extinctions across the Cretaceous/Tertiary boundary in planktonic foraminifera of continental-shelf sections: implications for impact and volcanism theories. Geol. Soc. Am. Bull., 101:1408-1419.

Kirschvink, J. L., 1980. The least-squares line and plane and analysis of paleomagnetic data. Geophys. J. R. Astron. Soc., 62:699-718.
Kyte, F. T., and Smit, J., 1986. Regional variations in spinel compositions: an important key to the Cretaceous/Tertiary event. Geology, 14:485-487.

Kyte, F. T., and Wasson, J. T., 1982. Geochemical constraints on the nature of large accretionary events. Spec. Pap. Geol. Soc. Am., 190:235-242.

1986. Accretion rate of extraterrestrial matter: iridium deposited 33 to 67 million years ago. Science, 232:1225-1230.

Michel, H. V., Asaro, F., Alvarez, W., and Alvarez, L. W., 1985. Elemental profile of iridium and other elements near the Cretaceous-Tertiary boundary in Hole 577B. In Heath, G. R., Burckle, L. H., et al., Init. Repts. DSDP, 86: Washington (U.S. Govt. Printing Office), 533-538.

Montanari, A., Hay, R. L., Alvarez, W., Alvarez, L. W., Asaro, F., Michel, H. V., and Smit, J., 1983. Spheroids at the Cretaceous/ Tertiary boundary are altered impact droplets of basaltic composition. Geology, 11:668-671.

Officer, C. B., and Drake, C. L., 1983. The Cretaceous-Tertiary Transition. Science, 219:1383-1390.

1985. Terminal Cretaceous environmental events. Science, 227:1161-1167.

O'Keefe, J. D., and Ahrens, T. J., 1982. The interaction of the Cretaceous-Tertiary extinction bolide with the atmosphere, ocean, and solid Earth. Spec. Pap. Geol. Soc. Am., 190:103-120.

Robin, E., Christophe Michel-Levy, N., Bourot-Denise, M., and Jéhanno, C., 1990. Crystalline micrometeorites from Greenland blue lakes: their chemical composition, mineralogy and possible origin. Earth Planet. Sci. Lett., 97:162-176.

Rocchia, R., Boclet, D., Bonté, P., Devineau, J., Jéhanno C., and Renard, M., 1987. Comparaison des distributions de I'iridium observées à la limite Crétacé-Tertiaire dans divers sites européens. Mem. Soc. Geol. Fr., N.S., 150:95-103.

Rocchia, R., Granier, B., Bellier, J.-P., Boclet, D., Bonté, P., Fourcade, E., Galbrun, B., Jéhanno, C., Lambert, B., Rasplus, L., and Renard, M., 1990. La limite Crétacé-Tertiaire du site de Finestrat (Province d'Alicante, Espagne). C. R. Acad. Sci. Ser. 2, 310:391-397.

Schmitt, R. A., 1989. Cometesimal explosions in Earth's atmosphere and steep angle cometesimal cratering events cause CretaceousTertiary extinction phenomena. Lunar Planet. Sci. Conf. 20th, 962-963. (Abstracts)

Shipboard Scientific Party, 1990. Site 761. In Haq, B. U., von Rad, U., O'Connell, S., et al., Proc. ODP, Init. Repts., 122: College Station, TX (Ocean Drilling Program), 161-211.

Smit, J., and Kyte, F. T., 1984. Siderophile-rich magnetic spheroids from the Cretaceous-Tertiary boundary in Umbria, Italy. Nature, 310:403-405.

Smit, J., and Romein, A.J.T., 1985. A sequence of events across the Cretaceous-Tertiary boundary. Earth Planet. Sci. Lett., 74:155170.

Timothy, D. H., and D'Hondt, S. L., 1990. Precessional climate cyclicity in Late Cretaceous-Early Tertiary marine sediments: a high resolution chronometer of Cretaceous-Tertiary boundary events. Earth Planet. Sci. Lett., 99:263-275.

Zachos, J. C., Arthur, M. A., Thunnell, R. C., Williams, D. F., Tappa, E. J., 1985. Stable isotope and trace element geochemistry of carbonate sediments across the Cretaceous/Tertiary boundary at Deep Sea Drilling Project Hole 577, Leg 86. In Heath, G. R., Burckle, L. H., et al., Init. Repts. DSDP, 86: Washington (U.S. Govt. Printing Office), 513-532.

Date of initial receipt: 30 April 1990

Date of acceptance: 15 January 1991

Ms 122B-179 

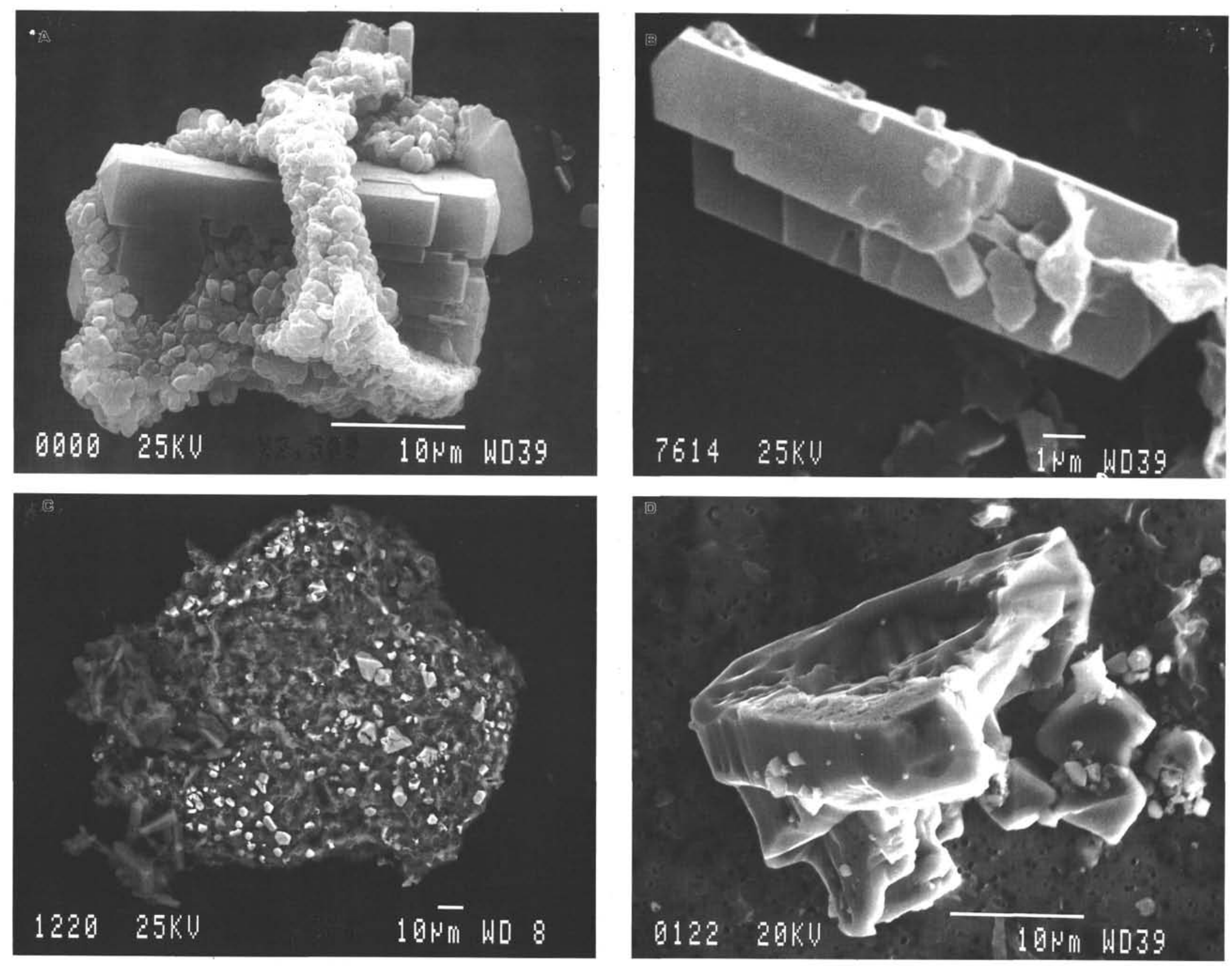

Plate 1. A. Clinoptilolite crystal that has grown inside a foraminifer test. B. Isolated clinoptilolite crystal. C. Magnetite-bearing flattened spheroid. Bright spots are magnetite crystals. D. Large Ni-rich magnetite. 